\title{
Échoendoscopie à la DDW, Nouvelle-Orléans, mai 2010
}

\author{
Endoscopic ultrasound at the DDW, New Orleans, May 2010
}

\section{B. Richard-Molard}

C) Springer-Verlag France 2010

Lors de la DDW 2010 à la Nouvelle-Orléans, de nombreux posters et quelques communications orales ont été consacrés à l'échoendoscopie (EE), surtout interventionnelle.

Dans le domaine diagnostique, plusieurs travaux ont confirmé l'intérêt de l'élastographie et de l'utilisation de produits de contraste pour caractériser la nature des tumeurs solides, avec une bonne valeur prédictive positive pour les tumeurs malignes et les tumeurs neuroendocrines [1,2]. Dans le domaine des TIPMP, l'EE est performante pour caractériser le type de TIPMP, du canal principal, des canaux secondaires ou mixtes, et aider à la décision thérapeutique $[3,4]$.

Le drainage par échoponction des voies biliaires par voie transgastrique ou transbulbaire avec pose de prothèse métallique couverte a fait l'objet de plusieurs travaux qui confirment la faisabilité de la technique et sa relative sécurité, en cas d'échec du drainage par CPRE [5,6]. Le drainage du canal pancréatique par prothèse métallique a également été présenté par une équipe, avec un taux de réussite de $93 \%$ [7]. Le drainage des pseudokystes pancréatiques réalisé avec des prothèses métalliques couvertes est l'objet de plusieurs travaux $[8,9]$, dont un rapporte une meilleure qualité de vie vis-à-vis de la chirurgie de drainage conventionnelle [10]. Quelques complications sont décrites : migration intrakystique de la prothèse, saignement contrôlé, pneumopéritoine contrôlé médicalement ; mais dans l'ensemble, la technique, si elle reste réservée à des centres tertiaires, semble efficace et sûre.

En conclusion, en ce qui concerne l'EE, rien de réellement innovant, mais la confirmation de l'extension croissante vers l'interventionnel.

\section{Références}

1. Ishikawa T, Hirooka Y, Itoh A, Kawashima H, Ohno E, Matsubara H, et al. T1446: Pancreatic endocrine tumors: preoperative localisation and differentiation of malignant versus benign by EUS combined with contrast-enhancement. Gastrointest Endosc 2010;71(5):AB279.

2. Badaoui A, Borbath I, Aouattah T, Gillain C, De Ronde T, Deprez P. T1455: evaluation of pancreatic tumors with contrast enhanced endoscopic ultrasonography and EUS-strain ratio elastography. Gastrointest Endosc 2010;71(5):AB281.

3. Ardengh JC, Ipolitto GD, Coelho Neto DE, Coelho JFE, Lima ER, Modena JLP, et al. T1433: MRCP versus EUS-FNA: what is the best way to diagnosis and classification (type and extent) of IPMT? Gastrointest Endosc 2010;71(5):AB276.

4. Harris CL, Malafa MP, Weber J, Klapman JB. T1469: the use of endoscopic ultrasound in differentiating side branch vs main duct vs mixed duct intraductal papillary mucinous neoplasm. Gastrointest Endosc 2010;71(5):AB285.

5. Park DH, Song TJ, Eum J, Moon SH, Lee SS, Seo DW, et al. T1482: clinical outcome of EUS-guided biliary drainage with a transluminal stent for biliary obstruction after a failed ERCP. Gastrointest Endosc 2010;71(5):AB288.

6. Fabbri C, Luigiano C, Polifemo AM, Ferrara F, Macchia S, Ghersi S, et al. T1435: EUS-guided biliary drainage for malignant biliary ducts obstruction: a prospective study. Gastrointest Endosc 2010;71(5):AB276.

7. Ergun M, Aouattah T, Gillain C, Piessevaux H, Gigot JF, Hubert C, et al. M1447: endoscopic ultrasound (EUS) guided transluminal drainage of pancreatic duct obstruction: long-term outcome. Gastrointest Endosc 2010;71(5):AB224.

8. Fabbri C, Luigiano C, Polifemo A, Ferrara F, Macchia S, Ghersi S, et al. T1434: endoscopic ultrasonography (EUS) drainage with self-expandable metallic stent (SEMS) in pancreatic pseudocyst (PP) and peripancreatic abscess (PPA): a prospective study. Gastrointest Endosc 2010;71(5):AB276.

9. Kim J, Shah JN, Marson F, Binmoeller KF. T1459: endoscopic drainage of pancreatic fluid collection with covered selfexpanding metal stents under EUS guidance. Gastrointest Endosc 2010;71(5):AB282.

10. Varadarajulu S, Trevino J, Wilcox C, Sutton B, Christein J. Randomized trial comparing EUS and surgery pseudocyst drainage. Gastrointest Endosc 2010;71(5):AB116. 